\title{
CONSIDERING THE HUMAN ACROSS LEVELS OF AUTOMATION: IMPLICATIONS FOR RELIANCE
}

\author{
Bobbie Seppelt ${ }^{1,2}$, Bryan Reimer ${ }^{2}$, Linda Angell $^{1}, \&$ Sean Seaman ${ }^{1}$ \\ ${ }^{1}$ Touchstone Evaluations, Inc. \\ Grosse Pointe, MI, USA \\ ${ }^{2}$ MIT AgeLab and New England University Transportation Center \\ Cambridge, MA, USA \\ Email: bseppelt@mit.edu
}

\begin{abstract}
Summary: This paper introduces human considerations that have yet to be fully addressed in industry standards for levels of automation. Currently-deployed vehicle automation is discussed according to these standards from a human interaction framing. The taxonomy-centric description of individual features provides insights into the challenges drivers may have in use of features in actual driving conditions. Initial data from an on-going naturalistic driving study of Tesla drivers is presented as a first-look at the prevalence of interaction challenges in real-world automation based on technology use. Implications for system design and training are discussed with the aim of centering industry and policy discussions on human-centric technology development.
\end{abstract}

\section{INTRODUCTION}

Automated vehicles are a topic of significant media and public discussion. Recent announcements on policy guidelines (NHTSA, 2016) and newly-released automated driving features (e.g., Tesla, Volvo, etc.) are illustrative of an evolving automobile marketplace. Industry and policy discussions center on a taxonomy of six levels of automation (SAE J3016, 2016) defined based on a binary allocation of driving subtask between the driver (or 'human') and system. This role division, in terms of who is assigned responsibility for the moment-to-moment lateral and longitudinal vehicle motion control activities, and for object event detection and response (OEDR) activities (collectively referred to as the dynamic driving task or DDT), differentiates Levels 0 - Level 3. The higher levels $4 \& 5$ are differentiated based on the operational design domain (ODD) - limited at Level 4, but unlimited at Level 5. According to J3016, the accurate description of an automation feature requires identifying both its level of driving automation (1-5) and its operational design domain (ODD). ODD refers to the conditions under which the automation is capable of performing its subtask(s), which may include "geographic, roadway, environmental, traffic, speed, and/or temporal limitations" (p. 12).

In the next section, this taxonomy, including the framing of human interaction, is considered in the context of a currently-deployed driving automation system to provide insight into the challenges drivers may have in use of driving automation features in actual driving conditions. Initial data from an on-going naturalistic driving study of Tesla drivers is next presented as a first-look into the prevalence of several interaction and training challenges in real-world advanced technology use. Finally, implications for system design and training are discussed in the context of future research, and the need to center industry and policy discussions on the human-technology dynamic. 


\section{Considering Level of Driving Automation from a Human-Centered Perspective}

Multiple automakers have released low-level automation features in commercially available vehicles (e.g. Tesla's Autosteer, Volvo's Pilot Assist, Mercedes-Benz's Drive Pilot, etc.). Table 1 lists and defines a subset of automation features that are available as part of one of these systems - the 2017 Autopilot Tech Package on Tesla Model S, and assigns to each a level of driving automation, applying the reference taxonomy (SAE J3016, 2016). Each feature is defined on the left-hand of Table 1, and, based on its described ability to execute sustained operation of either the longitudinal or lateral vehicle motion control tasks (Level 1), or both (Level 2), is classified into an automation level in the "Level - (based on system role alone)" column.

Table 1. Features Available in a 2017 Tesla Model S Based on Consideration of the System's Role and Based on the Combination of the Human and System Roles

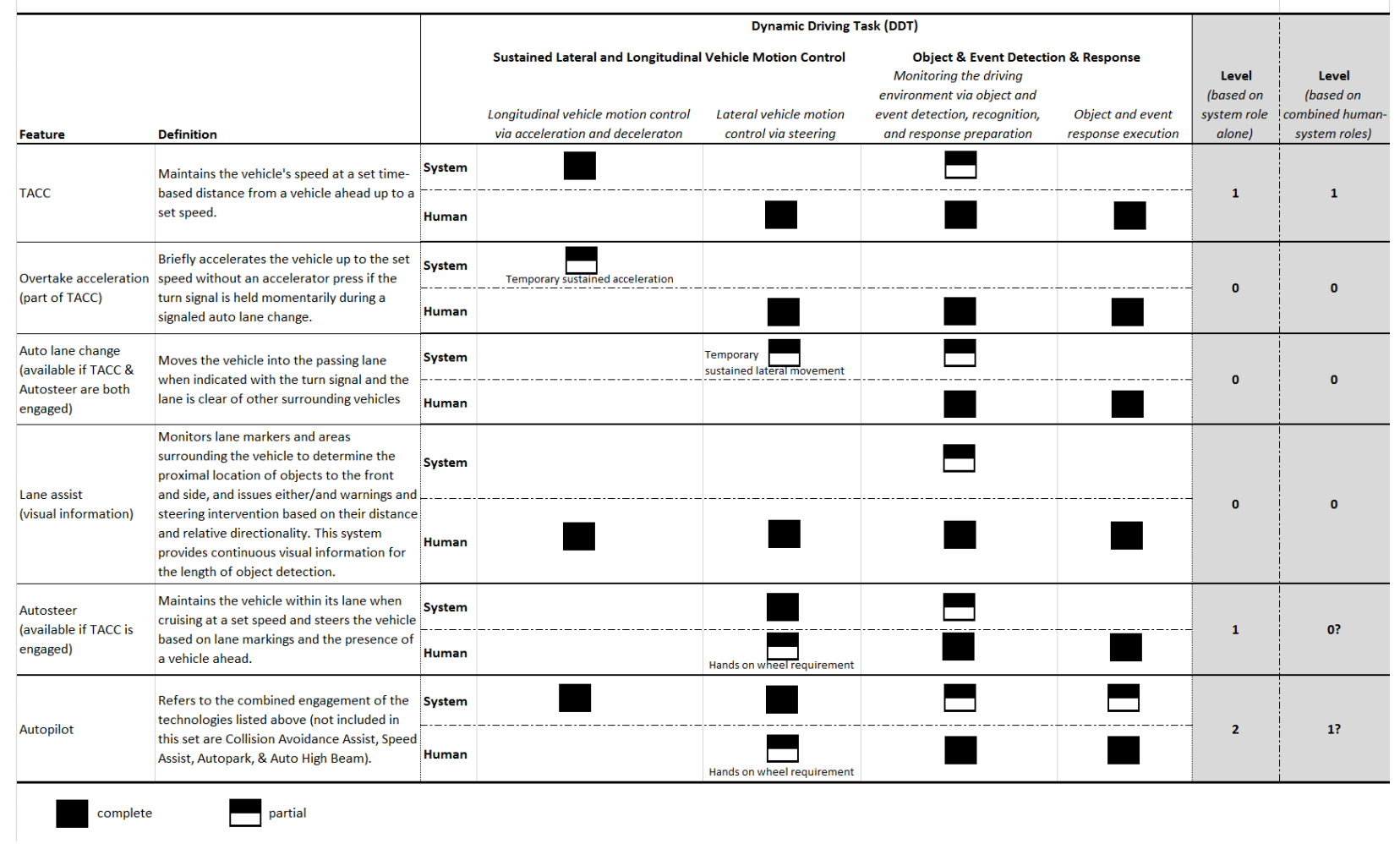

This system-centric classification is nominally consistent with the J3016 level definitions. New to this classification, complete and partial performance of a dynamic driving subtask are indicated using black and half-black boxes, respectively, to call out potentially meaningful differences not captured from a binary parsing of subtasks. Complete performance of a subtask refers to the SAE level definitions in a system's prescribed ability for active, sustained execution of a subtask. Partial performance is added to define instances when, though the subtask is not fully performed, it is partially supported based on the manual's described sensing functionality required to enable the fully-performed subtask functionality (e.g., camera or radar technology that detects moving objects in order to maintain a set distance to lead vehicle, or to visually display proximity of objects to the vehicle, etc.). The OEDR subtask of driving is broken out into its monitoring and response execution components to reflect instances when either monitoring the driving environment or executing a response may be completely or partially performed by 
either the human or the system (p. 12; SAE, 2016). Notably, only the subset of Autopilot's feature set that perform sustained DDT subtasks are included. When the human's role is considered in tandem in this way, the level distinction is called into question for Autosteer and Autopilot due to a hands-on-wheel requirement for the driver (right-most "Level" column). This requirement is an implementation-specific one. In use of Autosteer, a driver must keep his/her hands on the wheel and provide low-level steering input to keep this technology engaged; drivers will receive multiple warnings after a prolonged period of detected hands-off-wheel and eventually disengage. It is in this physical requirement for the driver to keep his/her hands on wheel and to provide some level of steering input in order to use this technology (cf. US Patent \#20140257628) that it is given a partially-filled box within the "Lateral vehicle motion control via steering" column for the driver. The "Level (based on combined human-system roles)" column brings into question the level definitions from a combined human-system role.

\section{Considering Operational Design Domain from a Human-Centered Perspective}

An operational design domain (ODD) defines, in practice, the set of conditions under which an automated feature or Advanced Driver Assistance System (ADAS) is designed for safe and reliable use. Based on implementation, an individual feature may be capable of performing single or multiple driving subtasks. Consequently, by level distinction, and dependent on the set of features engaged, ODDs may combine to create either a uniform or divergent set of appropriate use conditions.

For the same set of features referenced in Table 1, Figure 1 describes their speed-contingent ODD. The functional range for each technology is plotted on an individual line across a common speed range based on the feature's description within the owner's manual for the Tesla Model S, software versions 8 and 7 . Additional conditionality described within the manual per feature is noted above each line (e.g., if Autosteer is active vs. not active, the minimum functional speed for lane assist is 0 vs. 10). In comparing software versions 8 and 7, the functional speed range per feature and the associated set of operating conditions differs between versions. Figure 1 highlights the high degree of situation-dependency of those technologies that execute the lateral and longitudinal control tasks, as well as the amount of change between versions in how and when these features work. Beyond those shown for speed in Figure 1, derived from the sections within the manual that describe each feature, the operational conditions (or ODD) for which each feature is designed for use also vary based on factors such as the vehicle's position within a lane, road curvature, lane marking number and quality, lead vehicle presence and behavior, road type, and location of road features (i.e., tunnels, construction zones, tollbooths, and intersections). ODD variants within feature and across multiple features produce a complex array of use conditionality. 

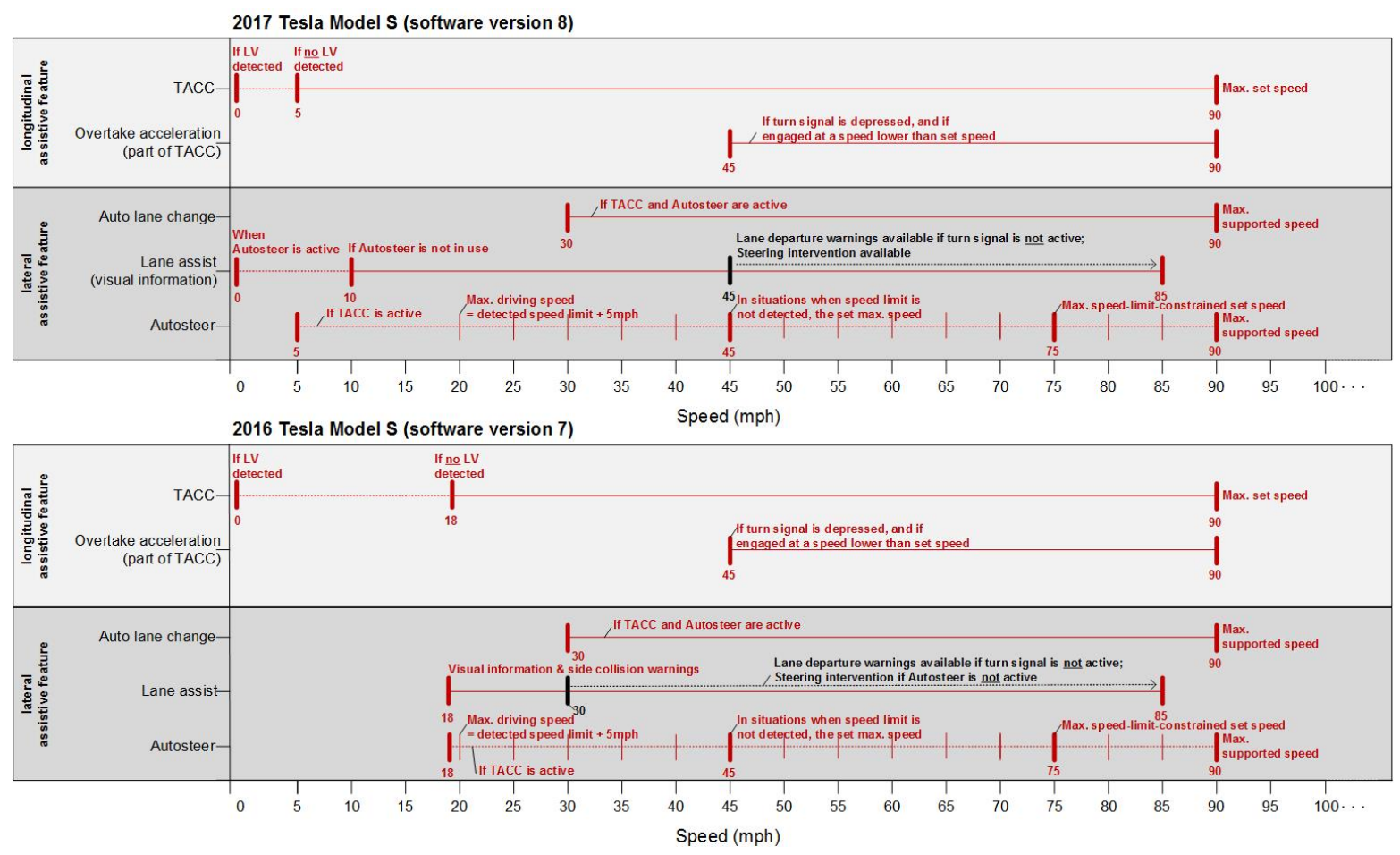

Figure 1. Available features on a Tesla Model S (software versions $7 \&$ 8) that assist the driver in sustained DDT subtasks. Features are grouped based on their assisted vehicle motion control directionality (in lighter grey box - longitudinal; in darker grey box - lateral). Each line shows an individual system's functional speed range. Additional conditionality is noted above each line. TACC = "Traffic-Aware Cruise Control"

At a high level, this review of feature ODDs revealed a potentially important difference between "static $O D D$ " and "dynamic $O D D$ " in a particular feature's conditionality. Static ODD refers to the set of environmental and roadway conditions with a fixed location and/or those that can be anticipated from knowledge of a particular route (e.g., entrance/exits to highways/interstates, start/end of construction zones, tunnels, and tollbooths, road type transitions, and intersections). Dynamic ODD refers to the set of environmental and roadway conditions that require on-board sensing to detect changes in state relative to vehicle position at a second-to-minute rate (e.g., lane marker visibility, presence of a LV, roadway curvature, etc.).

\section{Implications of Human-Centered Considerations on Reliance}

In applying the reference taxonomy to a currently-deployed set of automation features, a number of questions emerge that may have implications for driver reliance on these technologies in actual driving conditions.

Automation level - A need for drivers to understand their role vs. the system's role. The application of the reference taxonomy to a currently-deployed set of technologies reveals that, in practice, there is not a clean parsing of roles between driver and system. Individual features partially perform DDT subtasks redundant to the driver's role, calling into question if features with shared human-system performance fit into a clearly-prescribed level within existent taxonomies (e.g., SAE J3016, 2016). The hands-on-wheel requirement for the Autosteer feature is another instance of an unclear level delineation; the hands-on requirement (new to version 8) arguably shifts this feature from one that automates the lateral motion control task to one that assists the driver in this task. While a shared responsibility for steering movements may have a 
protective effect towards helping to keep drivers engaged at some level (Naujoks et al., 2015), this implementation constraint, in how its communicated to the driver, may place the driver at greater risk for inappropriate use (misuse - e.g., keeping hands off- wheel until warnings trigger - or disuse of this technology; Parasuraman \& Riley, 1997). Driver training and the adopted HMI strategy can help to calibrate a driver's expectations of the system and perceptions of system behavior (Endsley, 2017).

ODD - A need to match driver expectation to system capability. The look at an ODD for an example set of technologies available on a consumer vehicle reveals their high conditionality for recommended use. In feature combination, there is offset overlap in use conditions (e.g., variable speed range over which TACC works vs. TACC + Autosteer). An ODD, in defining the set of recommended use conditions for an automation feature (or set of features), specifies the appropriateness of a driver's decision to use this feature. Due to the high-specificity of ODDs, the driver may need to near-continuously monitor feature performance relative to use conditions to use them appropriately at their full capability, or, to practice high-level use decisions (e.g., only engage features on highways in daylight) that limit the potential benefits select technologies afford in other conditions. If an ODD is not understood/known or if a larger functional range is assumed than it was actually designed for, a driver is potentially at risk for inappropriate use (either over- or under- to recommended use) of a feature (or set of features). An automated driving system's ODD-specific execution of its subtasks may be important for a driver to know to make decisions as to when and for what set of subtasks s/he is responsible to perform vs. those of the automated system to use on-board technology at full capability.

An initial set of data from an on-going naturalistic driving study of Tesla Model S \& X drivers is presented to examine the extent to which the discussed use concerns are emerging from realworld driver behavior and experiences. It was hypothesized that drivers would use Autopilot in conditions outside of the recommended ODD due to its use conditionality in combination with limitations in user understanding of the systems (as guided by a wide variety of educational sources potentially developed for different versions of the system).

\section{METHODS}

A naturalistic driving study (NDS) is being conducted as part of a larger project exploring driver use of currently deployed advanced vehicle technologies at the Massachusetts Institute of Technology AgeLab. Over its 13-month enrollment period, over 100,000 miles of vehicle data have been collected on a fleet of 17 driver-owned Tesla Model S and X vehicles instrumented with in-cab and external video recording equipment and sensor suite (3 HD cameras, audio, CAN, GPS, accelerometer, and gyroscope data). Owners of Tesla vehicles from the greater Boston area have been enrolled in the study on a rolling recruitment starting in February 2016. An initial online questionnaire was administered in Fall 2016 to collect demographic information, details about their vehicle and its purchase, accessed training materials, and to probe perceptions and conditions of reported use for the Autopilot Tech Package. From this dataset, responses for a set of questions related to use of training materials and use conditions for the AutoPilot Tech Package were examined. This data provides an initial look at driver training on Autopilot as well as the prevalence of feature use outside of recommended ODD. 


\section{Participants}

Of the 17 participants enrolled in the study, four participants' survey data were removed due to partial questionnaire completion. The remaining set of 13 participants included in this initial analysis includes 12 males and one female with an age range from 21-75 (M=46.5, SD=15.2). The Tesla vehicles driven include seven Model S and six Model X from 2015-2016 model years. At the time of the initial survey, all 13 participants were driving with the Autopilot Tech Package on-board and reported accepting software version updates.

\section{Procedure}

The initial questionnaire included a set of six demographic questions, eight questions on the particular vehicle model and on-board technologies at point of purchase, and nine questions on perceptions and use conditions of the Autopilot Tech Package, for a total of 23 questions.

\section{RESULTS}

Two questions that specifically addressed Autopilot training and use were subset from the total set of 23. These questions were: 1) How did you learn to use the Autopilot system in your vehicle? and 2) In what driving conditions do you frequently engage Autopilot?. Counts for selections of the two multi-option questions are shown in Figure 2 below.
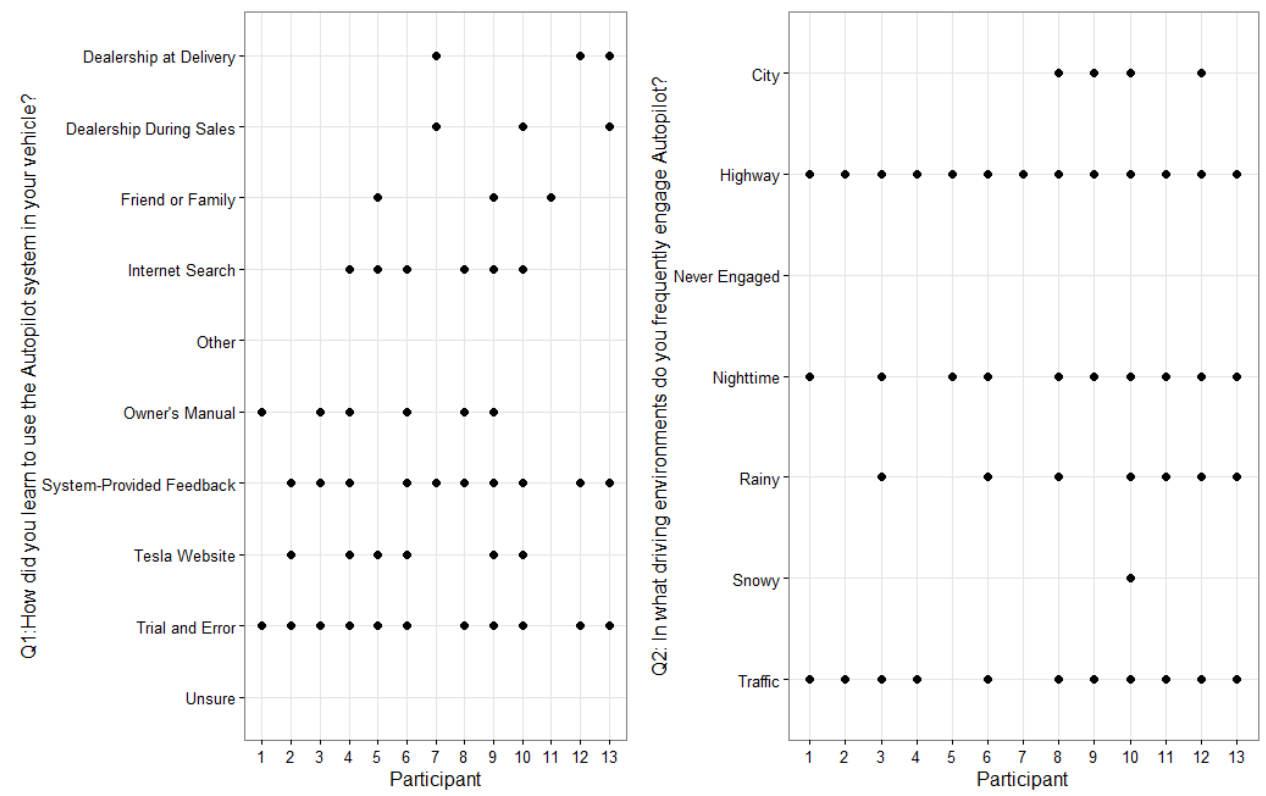

Figure 2. Counts of participants' responses per option for two use questions

For the question on types of learning, all drivers reported multiple methods of learning $(\mathrm{M}=3.7$, $\mathrm{SE}=0.38$ ). For accessing the detailed information on ODDs found within the owner's manual, 6 out of 13 drivers reported learning about Autopilot through this method (6 out of 13). For the question on conditions of Autopilot engagement, a total of 7 out of 13 drivers reported using the set of technologies in rainy or snowy conditions - conditions specifically noted within the owner's manual as factors that adversely impact Autopilot performance. 


\section{CONCLUSIONS}

The first part of this paper examines human considerations for the industry-standard level distinctions for a set of automated features and their ODD on-board a commercially available vehicle. This review revealed concerns over driver use of these features due to potentially misunderstood role distinctions complicated with a confusing array of ODD. Initial self-report data from a larger NDS dataset indicates that some drivers are engaging systems in conditions outside of recommended ODDs. Access to available materials on system technologies does not preclude this behavior. Drivers may be aware of feature ODDs and willfully deciding to engage them outside of recommended use conditions or in need of more information on when and how to use features to use them appropriately. The proposed classification of ODDs into "static" and "dynamic" elements may have implications for driver understanding of ODD boundaries relevant for in-vehicle feature displays and driver training strategies. Planned as a next step, a comparison of the reported subjective NDS data to use statistics for the set of conditions in which drivers use automated features should help to disentangle this relationship. Overall, the highlighted issues point to the emerging need to specify operational conditions at a level consumers can intuitively understand or learn by aid of on-/off-line training approaches (manuals, driver coaching, etc.). Automakers may alternatively need to design systems with less constrained or common cross-feature ODD.

\section{ACKNOWLEDGEMENTS}

Support for this work was provided by the US DOT's Region I New England University Transportation Center at MIT and the Toyota Class Action Settlement Safety Research and Education Program. The views and conclusions being expressed are those of the authors, and have not been sponsored, approved, or endorsed by Toyota or plaintiffs' class counsel. Data was collected through efforts supported by the Advanced Vehicle Technology (AVT) Consortium.

\section{REFERENCES}

Endsley, M. R. (2017). Autonomous Driving Systems: A Preliminary Naturalistic Study of the Tesla Model S. Journal of Cognitive Engineering and Decision Making, 1555343417695197.

Lee, J.-W., Litkouhi, B., \& Huang, H.-H. Steering-wheel-hold detection for lane keeping assist feature. U.S. Patent 20140257628. Sept. 11, 2014.

Naujoks, F., Purucker, C., Neukum, A., Wolter, S. \& Steiger, R. (2015). Controllability of partially automated driving functions - does it matter whether drivers are allowed to take their hands off the steering wheel? Transportation Research Part F, 35, 185-198.

Parasuraman, R., \& Riley, V. (1997). Humans and automation: Use, misuse, disuse, abuse. Human Factors: The Journal of the Human Factors and Ergonomics Society, 39(2), 230-253.

SAE, Taxonomy and Definitions for Terms Related to Driving Automation Systems for OnRoad Motor Vehicles, SAE Standard J3016, USA, 2016. 